http://doi.org/10.15359/ree.12-2.8

\title{
FREIRE: VIGENTE E INSPIRADOR EN LA ALFABETIZACIÓN DE PERSONAS ADULTAS
}

\author{
Roberth Barboza Araya ${ }^{1}$ \\ Estudiante de Bachillerato en la Universidad Nacional \\ Heredia, Costa Rica \\ Samantha Camacho Varela ${ }^{2}$ \\ Funcionaria del Instituto Costarricense de Electricidad, ICE \\ San José, Costa Rica \\ Luz Emilia Flores Davis ${ }^{3}$ \\ Docente, investigadora y extensionista en la Universidad Nacional \\ Heredia, Costa Rica \\ Ana María Hernández Segura ${ }^{4}$ \\ Docente, investigadora y extensionista en la Universidad Nacional \\ Heredia, Costa Rica \\ María Goretti Vargas Alpízar ${ }^{5}$ \\ Estudiante de Educación Especial en la Universidad Nacional \\ Heredia, Costa Rica \\ Jeimmy Venegas Ugalde \\ Estudiante de Educación Especial en la Universidad Nacional \\ Heredia, Costa Rica
}

Recibido: 7 de marzo, 2008 • Aprobado: 15 de mayo, 2008

Resumen: Este artículo presenta diversas experiencias en torno a la alfabetización de personas adultas, inspiradas en el pensamiento de Paulo Freire. La vigencia de los aportes de

Diplomado en Pedagogía con Énfasis en I y II ciclos de la Educación General Básica. Actualmente es estudiante de Bachillerato en la misma carrera en la Universidad Nacional. rbary07@yahoo.es

Profesional en Planificación Económica y Social con Especialidad en diagnóstico de necesidades y problemas sociales. Consultora e investigadora en el área de Impacto Ambiental. Funcionaria del Instituto Costarricense de Electricidad, ICE. scamachov@ice.go.cr

Máster en Educación Superior y catedrática universitaria. Actualmente se desempeña como docente, investigadora y extensionista en la Universidad Nacional. luzdeflores@yahoo.com

Máster en Educación, con Mención en Docencia Universitaria, Licenciada en Educación Preescolar y Terapista de Lenguaje. Actualmente se desempeña como docente, investigadora y extensionista en la Universidad Nacional. anahernandez63@hotmail.com

Diplomada en Pedagogía con énfasis en I y II ciclos y Bachiller en Educación Especial con énfasis en Integración. Universidad Nacional. Actualmente cursa la Licenciatura en la misma carrera. mgva1908@yahoo.com

6 Diplomada en Pedagogía con énfasis en I y II ciclos y Bachiller en Educación Especial con énfasis en Integración. Universidad Nacional. Actualmente cursa la Licenciatura en la misma carrera. jeimmyvu@hotmail.com 
Freire son reconstruidos mediante un trabajo académico integrado, llevado a cabo en diferentes contextos, mediante la coordinación interinstitucional entre la Universidad Nacional y el Instituto Costarricense de Electricidad, con la participación de profesionales y estudiantes.

Palabras clave: Alfabetización, lectura, escritura, personas adultas.

\begin{abstract}
This article presents diverse experiences regarding alphabetization of grown-ups, inspired by Paulo Freire's ideas. The validity of Freire's contributions are reconstructed through an integrated academic work, carried out in different contexts, through the coordination between the National University (Universidad Nacional) and the Costa Rica Electricity Board (Instituto Costarricense de Electricidad), with the aid of professionals and students.
\end{abstract}

Key words: Alphabetization, reading, writing, adults.

\title{
Introducción
}

Retomar los sueños, reencantar la educación, abrir nuevos horizontes, eso nos inspira la obra de Paulo Freire. Su compromiso con la democracia, y, por tanto, con los sectores de la sociedad que no tienen voz, infunde fuerzas que trascienden el tiempo y el espacio, y se convierte en potente gatillador de acciones reflexivas para transformar la educación y las formas de convivencia.

El legado de Paulo Freire nos reúne hoy. Aproxima personas e instituciones que trabajamos en torno a la construcción de una sociedad más equitativa y respetuosa de la diversidad. El intercambio de experiencias y la posibilidad de establecer verdaderos diálogos, nos permiten profundizar nuestras reflexiones en torno a la educación actual y la que soñamos.

Entusiasmo y esperanza, son herencias de Freire que necesita hoy, más que nunca, la educación. Una educación liberadora que fundamente una pedagogía orientada por principios de participación, solidaridad y autonomía. Una pedagogía con la que las personas seamos cada vez más humanas, y estemos comprometidas con el cuidado del Planeta.

El coraje de poner en práctica una pedagogía de la pregunta, una pedagogía de la autonomía, una pedagogía de la esperanza; un auténtico esfuerzo por la alfabetización en el más amplio sentido de la palabra, precisa procesos de concienciación en todos los niveles del sistema educativo. 
Freire nos ha legado una pedagogía crítica con visión esperanzadora que entiende la educación como copartícipe en el cambio social. En este sentido, revaloriza y resignifica la enseñanza como pilar de la relación educativa. Desde su concepción, la enseñanza trasciende la transmisión de conocimientos, para formar, para mediar en la construcción de saberes contextualizados.

Freire vive la indignación contra todo tipo de injusticia, y su obra instaura una relación inquebrantable entre educación y política y, por tanto, entre pedagogía y poder.

Queremos compartir, en esta ocasión, nuestras experiencias y reflexiones respecto al poder que tiene la palabra, la palabra de la persona no alfabetizada en el siglo XXI, y la palabra de estudiantes y profesionales que viven el compromiso con una práctica democrática de la educación.

Seguidamente, se presentan tres experiencias de alfabetización realizadas por distintas personas y en distintos contextos, pero todas ellas en el marco de un proyecto de Lectura y escritura de la División de Educación Básica del Centro de Investigación y Docencia en Educación (CIDE), de la Universidad Nacional de Costa Rica (UNA), con el apoyo de las docentes responsables del proyecto. Las tres experiencias se inspiran en las ideas propuestas por Freire, en la necesidad de una reflexión crítica sobre la práctica, de vivir la educación con alegría y esperanza, y respetar los saberes de los educandos.

\section{Concluir viejas metas y trazar nuevos retos}

La primera de las experiencias que se presenta fue realizada por uno de los coautores de este artículo, Roberth Barboza, cuando era estudiante de primer nivel de la carrera de I y II Ciclos de la Educación General Básica, quien llevó a cabo una práctica de alfabetización como parte de su formación docente, con una señora de su comunidad, Puriscal, quien comparte su vivencia por medio del siguiente relato:

Nos encontramos en la época denominada de la información y el conocimiento global, en la que la vida de la mayoría de las personas, se encuentra rodeada por grandes volúmenes de 
información que llega hasta nuestras manos, gracias a los medios de comunicación masiva, como es el caso de la televisión, el periódico y la Internet. Se supone que, en esta época, todas las personas saben leer y escribir, y, por tanto, el interés se enfoca en estar actualizado en el manejo de las nuevas estrategias de comunicación y administración de datos.

Pero, más cerca de lo que pensamos, se encuentra una realidad muy diferente, realidad que, diariamente, tienen que enfrentar muchos hombres y mujeres, cuya preocupación no es tener acceso a lo último en información, sino que su reto lo constituye el recordar el color del autobús en que salieron de un lugar para regresar en el mismo, ingeniárselas para distinguir entre la pastilla para aliviar el dolor de cabeza o para dormir, esto porque no pueden leer los rótulos, las descripciones, o las recetas que les permitan acceder a la información que allí se presenta.

Las situaciones anteriormente expuestas son algunas de las razones que dan origen a la experiencia vivida al alfabetizar a una persona adulta. Según el investigador, en un primer momento se le planteó como un gran reto, ya que se encontraba en su primer año de formación como docente, por tanto, no se sentía plenamente capacitado para lograr con éxito la tarea propuesta. Además, como se mencionó al inicio, creía que, actualmente, sería muy difícil encontrar a una persona que no supiera leer y escribir. Sin embargo se dio a la tarea de encontrarla, y efectivamente, lo hizo y más cerca de lo que se imaginaba.

En el primer acercamiento no hubo una respuesta positiva ante el ofrecimiento, ya que después de tanto tiempo, Adela ${ }^{7}$ había logrado crear estrategias para tratar de solventar la situación, creyendo que a sus 45 años ya no era necesario aprender. Sin embargo, existía la inquietud por independizarse en este campo y no depender de los demás, ya que eran otros los que tenían que darle la información respecto al producto o servicio que necesitaba. A partir de ahí, Adela podía generar diferentes estrategias que le permitieran clasificar o ubicar lo que necesitaba con mayor facilidad. Según lo que el investigador pudo indagar en su momento,

7 Los nombres que aquí aparecen no corresponden a los verdaderos, sino que se han utilizado códigos, o sea, nombres inventados 
dichas asociaciones se realizaban gracias al color, forma, memorización de la palabra como una imagen, entre otras.

El tema de la alfabetización de personas adultas es muy complejo, y la mejor forma que encontró para conocer las vivencias y la realidad de una persona en estas condiciones, fue acercarse a ella, demostrarle que el hecho de no estar alfabetizada no la hace inferior a nadie, motivarla para que expresara las experiencias que había tenido que enfrentar, por no saber leer y escribir; interesarse por la historia que ofrecía, las anécdotas de su niñez y juventud, entre otros aspectos que le permitieran tener un contacto más cercano con su realidad, experiencias e historia de vida, ya que consideró que, como docente, no se pueden llevar a cabo procesos de enseñanza y de aprendizaje, sin conocer a la persona con quien se va a compartir.

Asimismo, el tener la información anterior le permitió utilizar algunas de sus experiencias como motivación para que fuera modificando su autoconcepto, ya que la participante en este proceso de aprendizaje, estaba muy convencida de que ella nunca aprendería, aunque se sintiese mal por no saber leer y escribir; después de tantos años se repetía: "Soy muy tonta y nada se me pega". Por consiguiente, como se mencionó, Adela no se mostraba motivada, principalmente, porque creía que no tenía la capacidad para aprender.

Por esto, debe tratarse, primero, de hacer cambiar la vieja mentalidad, especialmente, por medio de diferentes experiencias que le demuestren, de forma concisa, y con hechos evidentes, que sí puede aprender y mejorar su condición actual. Otro aspecto que el alfabetizador consideró necesario fue hacerle comprender a Adela que el aprendizaje debía ser entendido como una meta personal para sentirse mejor, y, además, darse cuenta que, por sí misma, podría lograr otros propósitos. Además, era necesario crear una conciencia de competencia consigo misma, reconociendo que cometemos errores, porque ésta es la característica que nos recuerda diariamente que somos seres humanos y que, como tales, acertamos en algunos aspectos y erramos en otros. Estos últimos deben ser tomados en cuenta para enriquecer nuestra experiencia, y poder incorporarlos, satisfactoriamente, en las actividades diarias.

Superadas estas primeras etapas de acercamiento, conocimiento y planteamiento del proceso por seguir, se inició, sin dejar de lado, en ningún momento, el crecimiento en cuanto a 
autoestima, lo que es indispensable para fortalecer la tarea del aprendizaje, y que debe ser trabajada de forma transversal en el proceso.

Al iniciar esta tarea hay que comprender que la persona adulta es muy diferente a los niños y las niñas y, como tal, tiene una perspectiva distinta del mundo que le rodea; en este caso, en particular, es negativa, por todos los acontecimientos que le han marcado y le han ayudado a formar la realidad en la que vive. A lo anterior, se suman múltiples preocupaciones, principalmente económicas y de vivienda, que marcan las prioridades que se tienen.

Un proceso pedagógico no estaba dentro de sus planes, por tanto, era un gran reto mantener vivo ese deseo de superación, lo que demandaba un mayor grado de dedicación y análisis de la práctica que se desarrollaba minuto a minuto.

Para tener éxito en el proceso de alfabetización deben proponerse alternativas llamativas que respondan a sus intereses, perspectivas y necesidades. Por esta razón, hay que estar reflexionando, constantemente, sobre aquello que surgió durante la conversación entablada, preguntarse por qué se dio esa situación y cómo puede incorporarse en el proceso. No está de más evaluar la práctica de enseñanza como tal, y dentro ella, la labor que, como docente, se está cumpliendo. Pero estos momentos de constante reflexión y análisis de todas estas situaciones, no deben desperdiciarse, sino que deben ser la base para nuevos conocimientos que permitan tomar decisiones acordes con la realidad del proceso desarrollado. Estas decisiones van a estar dirigidas a mejorar la propuesta que cada día se desarrolla.

De lo anterior se desprende el hecho de que un proceso unidireccional no cabe dentro de la alfabetización de personas adultas. Para que, realmente, se logren los objetivos esperados debe realizarse un trabajo de equipo, en el que tanto el docente como el discente aporten lo que saben.

En un principio, esta experiencia se inició con el método para la enseñanza de la lectura y escritura del Nombre propio, el cual, como su título lo indica, parte del nombre de la persona a la que se le desea enseñar, tomando en cuenta que éste es significativo para ella y que la identifica. Posteriormente, y de acuerdo con los resultados del análisis y la reflexión de la práctica, se orientó el proceso hacía la incorporación de otros elementos ligados a sus experiencias e intereses y uno de esos materiales fue un periódico de circulación local llamado 
El Puriscaleño; con el que se desarrollaron diferentes actividades, a partir de las cuales se logró conocer un poco más sobre el cantón, lo que permitió recordar diferentes lugares y, al mismo tiempo, contrastar lo que se revive con la imagen que se encuentra en el documento en cuanto a paisajes, construcciones, personajes, anécdotas de lugares, entre muchos otros aspectos.

Así, a partir de lo expuesto, cada día fue posible ir variando las actividades, los elementos y las estrategias, de forma que se fueran cumpliendo varios de los objetivos que se iban estableciendo. Aquellos que, por alguna razón, no se plasmaban, se replanteaban para ser retomados en nuevas oportunidades, tratando de incorporar aquello que, en primera instancia, faltó o sobró, y si al reflexionar el docente se daba cuenta de que no cabía dentro del proceso, lo remplazaba por otro que sí correspondía con esa realidad.

Al concluir la experiencia se evidenciaron muchos cambios, principalmente, en cuanto al autoconcepto de la discente y al desempeño que puede tener en la sociedad. La reestructuración de las ideas sobre sí misma abrió las puertas para que Adela se diera la oportunidad de aprender y desarrollar habilidades como las siguientes: ser capaz de escribir oraciones y párrafos, expresarse oralmente con mayor claridad y fluidez, ser capaz de leer, entender y criticar el mensaje del texto, entre otros aspectos. Además, como ella misma lo indicó en algún momento, se siente más segura de lo que hace, toma mayor cantidad de decisiones por sí misma. En cuanto a esto último, dio un paso que para ella fue trascendental, ya que solicitó el Bono de la vivienda, y ella misma asumió la responsabilidad de efectuar todo el papeleo que implica este trámite, y, gracias a la decisión que tomó, hoy tiene su casa propia.

Recordemos que el docente debe asumir y reestructurar nuevos parámetros que rijan su práctica. En esta experiencia, se trató de implementar una de las frases célebres que plantea Freire (1998), la cual dice: "Enseñar exige reflexión crítica sobre la práctica" (p.39), reflexión que nos llevará a ahondar en cada una de aquellas situaciones que, tal vez, consideramos poco significativas, pero al ser sometidas al cuestionamiento y al análisis, permiten encontrar el hilo conector entre cada una de ellas, resultando, en la mayoría de los casos, sorprendentes.

Precisamente, esto fue lo que sucedió cuando el profesor empezó a descubrir que más que un proceso pedagógico estructurado y dirigido por él, lo que se debía desarrollar era una serie de experiencias y situaciones significativas para la aprendiz. 
Como se evidencia en el proceso descrito, hay una gran inclinación a la oralidad (acontecimientos diarios, historias casi increíbles, recuerdos que han estado guardados por años, costumbres ya olvidadas, forma de vida) y el desarrollo y la consolidación de la parte humana, elementos que permiten el crecimiento personal. El alfabetizador considera que el darse cuenta de esos dos aspectos fue un gran paso, que al mismo tiempo lo impulsaban a trazar nuevos retos que, como docente, debe tener siempre presentes, para así ofrecer, a diario, lo mejor. Queda en las manos de cada uno seguir construyendo esperanzas que, a su vez, darán paso a la culminación de metas postergadas y al establecimiento de algunas nuevas.

\section{Vivir la alegría y la esperanza}

La segunda experiencia de alfabetización que se comparte fue llevada a cabo por dos de las coautoras de este texto, María Goretti Vargas y Jeimmy Venegas, cuando eran estudiantes de la carrera de Educación Especial, quienes escogieron la alfabetización de un joven con retardo mental, a quien llamaremos Luis, como tema para elaborar su tesis de Licenciatura.

Con el fin de caracterizar un poco más al aprendiz, es importante mencionar que él es un hombre de veinticinco años de edad, el cual presenta una discapacidad de tipo cognitiva, y debido a que en su etapa escolar no se dio énfasis al aprendizaje de la lectoescritura, es que se retoma en este momento.

Comprender el entorno en el que se vive, interactuar con él y desenvolverse sin temor e inseguridad, ha de ser parte de todo ser humano. Esta convivencia con el contexto constituye un derecho fundamental del que deberían disfrutar todo hombre y toda mujer, y la educación es uno de los instrumentos que proporciona los conocimientos y las habilidades para lograr una adecuada adaptación al medio social en el cual corresponde vivir.

La educación es un derecho universal, que involucra a las personas sin importar sexo, raza, credo, condición socioeconómica o diferentes capacidades. La adquisición de conocimientos, tanto académicos como funcionales que se logre, dentro o fuera de los centros 
educativos, va a repercutir, favorablemente, en el desenvolvimiento de las personas, lo que les permitirá, con el tiempo, la movilidad social y, por ende, una mejor calidad de vida.

Si bien es cierto, teóricamente, esta concepción pareciera ser la más atinada; en la realidad, se viven situaciones que la hacen ver como una utopía. Los grupos minoritarios, tales como los inmigrantes, quienes viven en extrema pobreza o presentan algún tipo de condición discapacitante, se convierten en los más vulnerables para quedar excluidos del derecho a un adecuado proceso de aprendizaje.

El hecho de aprender a conducirse en el ambiente, comunicarse asertivamente, resolver problemas cotidianos, entablar y mantener relaciones con las demás personas, y hacer uso de la lectura y la escritura, hacen referencia a habilidades básicas que propicia una alfabetización funcional.

La alfabetización es un acto de comprensión, un recurso que permite al ser humano expresar sus ideas, sentimientos y emociones; a la vez, que le facilita recibir e intercambiar información con otras personas. Es, también, un medio que fortalece la autoestima y facilita los recursos para el desarrollo de la independencia y, por ende, la autorrealización.

Generalmente, se piensa que la alfabetización es un proceso que se da, de manera exclusiva, durante la infancia y dentro de una institución educativa. No obstante, existen personas adultas que, por diversas razones, no han tenido la oportunidad de participar en una experiencia alfabetizadora. Por tanto, para una persona adulta, con el sueño de aprender y con las ansias de alcanzar deseos que se veían truncados por los escasos recursos de lectoescritura y de acceder a un proceso de alfabetización institucionalizado o no, se convierte, muchas veces, en una fuente de alegría y motivación, pues le devuelve la esperanza de luchar por los anhelos que antes se encontraban obstaculizados.

Además, aunado a la esperanza que albergan las personas por aprender, se encuentra, también, el deseo por enseñar que ha de tener el educador o la educadora. Todo aprendizaje está estrechamente ligado a la motivación, tanto de quien aprende como de quien enseña. La esperanza de que la otra persona puede aprender, de que sea capaz de superarse, debe ser el eje del que se parta, pues no tendría lógica enseñar e invertir tiempo en acciones que no van a tener resultados, por mínimos que sean los alcances. 
Así mismo, la metodología que se emplee en la construcción del conocimiento debe tener en cuenta los intereses, las limitaciones, las necesidades y las características de quienes están involucrados, ya que, de esta manera, será significativo y habrá mayores probabilidades de alcanzar el éxito. Es necesario que las actividades metodológicas que se planteen se ajusten a cada participante, que tengan como fundamento el entorno y partan de lo que motivó a la persona. Sabemos que aprender a leer y a escribir no es una tarea sencilla, pero la buena mediación docente y la motivación, constituyen factores que facilitan y promueven el proceso. A esto hay que agregarle una dosis de alegría y otra de esperanza, para que, unidas, surtan efectos positivos en la vida de las personas, pues estos elementos son directamente proporcionales al aprendizaje y se ven reflejados en las actitudes de los participantes.

De igual manera, para una persona adulta con discapacidad cognitiva, todos estos aspectos requeridos para la alfabetización, deben tener aún mayor peso. Las estrategias por utilizar deben estar acordes con sus limitaciones y las posibilidades que le ofrece el entorno. Además, deben considerarse las expectativas reales que tienen el participante y el mediador, a fin de que haya ilusión y ánimo a lo largo del proceso de aprendizaje.

Las actividades desarrolladas en esta experiencia de alfabetización incluyeron momentos para conversar, practicar la lectura y la escritura con vocabulario de diferentes contextos con los que tiene contacto la persona que aprende, como por ejemplo: la casa en la que habita, la pulpería, los restaurantes, la librería, los medios de transporte, así como los lugares de recreación que utilizan las personas de su edad, entre ellos el centro comercial, el cine, las heladerías, el parque.

Estas dinámicas de trabajo han generado un gran aporte en el área académico-funcional y en la parte afectiva, porque se han visto resultados positivos en el aprendizaje de la lectura y la escritura, en el desenvolvimiento dentro del entorno, en la interacción con otras personas y hasta en el fortalecimiento de la autonomía, la independencia, la seguridad y, por ende, de la autoestima.

Actualmente, y gracias al trabajo conjunto con la familia, es posible que Luis desempeñe un oficio en un negocio familiar: una verdulería. Ahí labora y realiza diferentes funciones, tales 
como atender clientes, pesar productos, acomodar la mercadería y estar pendiente del vencimiento de las frutas y las verduras.

Esta verdulería ha sido un medio por el cual muchas personas se han visto beneficiadas de distintas maneras, ya que aparte de generar un ingreso económico a la familia, el participante en proceso de alfabetización mantiene una mayor interacción con otras personas, pone en práctica sus conocimientos, se le asignan responsabilidades, cumple un rol importante dentro de su núcleo familiar, comprende el entorno que le rodea, continúa aprendiendo y le produce satisfacción personal.

Por tanto, la alfabetización como fuente de esperanza y alegría, es un proceso que debe estar al alcance de todos, proveyendo herramientas para la autonomía, convirtiéndose, así, en un instrumento elemental para la vida; con más razón, en la población con necesidades educativas especiales, pues, también, son parte de la sociedad, capaces de desempeñar papeles que anulen las diferencias que la misma comunidad genera, y la alfabetización proporciona un sentimiento de pertenencia y de satisfacción.

\section{Los saberes de los educandos}

La tercera vivencia corresponde a una experiencia que se realizó como un esfuerzo conjunto entre el Instituto Costarricense de Electricidad ICE y la Universidad Nacional. Fue llevada a cabo por una de las coautoras de este artículo, Samantha Camacho, quien es profesional en Planificación Económica y Social, funcionaria del Proyecto Hidroeléctrico Cariblanco del ICE, como parte de las medidas de compensación social indirecta, en una de las comunidades del área de influencia directa del proyecto, quien participó, para ello, en una serie de talleres efectuados en el marco del Proyecto de lectura y escritura, dirigidos a personas voluntarias, interesadas en alfabetizar en sus comunidades. La vivencia fue realizada en Virgen del Socorro de Sarapiquí de Heredia.

En Costa Rica, la educación es libre, gratuita y obligatoria; es un beneficio al que todos tenemos derecho; sin embargo, las oportunidades de acceso no son iguales para todos. El 
analfabetismo limita y disminuye los espacios participativos de la población que se encuentra en esa condición. El promover procesos de alfabetización en las comunidades menos privilegiadas representa un aporte para su mejoramiento en la calidad de vida.

Con el fin de contribuir a disminuir esta brecha en educación se promovió un proceso de alfabetización con personas adultas en la comunidad antes mencionada, específicamente con cinco miembros de una familia: Ana, Pedro, Manuel, Jorge y Gerardo, con edades entre 20 y 28 años, Este proceso se enfoca bajo las orientaciones de Freire (1998). La frase guía durante la alfabetización fue: "Enseñar exige respeto a los saberes de los educandos" (p. 31). Así pues, este trabajo se inicia fomentando métodos de aprendizaje enfocados hacia el respeto y la valoración de los educandos.

El proceso de aprendizaje implicó conocer a los participantes y saber cuál era su sentir con respecto a su vida cotidiana. El conocimiento de su entorno refleja el interés que tenemos respecto a su individualidad. Por esta razón, se inició el trabajo de alfabetización conociendo y anotando su nombre en un papel, lo que permite valorar cada nombre como propio y único. La autoestima fue un tema constante en el transcurso de las sesiones.

Es importante tener presente que debe ser el facilitador quien tiene que readecuar sus horarios, de acuerdo con el de los educandos. En este caso concreto, los miembros de la familia con los que la facilitadora tuvo la oportunidad de compartir durante ocho meses, fueron quienes decidieron sus horarios. Para ellos, este proceso educativo fue muy importante, esto se reflejó en su formalidad al vestir para participar en las lecciones, pues asistieron con ropa muy formal; para ellos representó una ocasión muy importante en la semana.

Las sesiones se desarrollaron en el aula de la escuela. En la primera sesión no querían ingresar en el aula, se sentían apenados. Conforme avanzaban las reuniones con el grupo, se mostraban más cómodos. En ocasiones, se desarrollaron las clases fuera del aula, para cambiar de ambiente y propiciar el interés por otros medios. Se decidió con el grupo trabajar una vez por semana: los martes de 6 p.m. a 8 p.m. El proceso se registró de manera individual, porque cada educando es diferente y, por tanto, sus capacidades difieren también.

Es importante anotar que el respeto incluye la importancia que cada facilitador le dé a su trabajo, es decir, la seriedad con que se asuma un reto tan significativo como lo es la 
alfabetización a adultos, y la seriedad con que realiza la preparación previa de material para el trabajo en cada sesión, la cual debe ajustarse a los discentes.

Generar la confianza en los participantes es un aspecto básico en el papel del facilitador, escuchar y ser escuchados permite crear un ambiente de confianza. Conocer su percepción de vida es vital para despertar el interés de la persona durante el período de trabajo. Se valoran sus intereses y, a partir de ese punto, se inicia la labor con cada uno.

Los intereses de los actuantes son distintos; Ana, la única mujer participante, se emociona cuando logra escribir oraciones sobre sus hijos. Según expresó, al inicio del proceso, le interesaba aprender a leer y a escribir para enseñarles. Ahora, ayuda a sus hijos con los trabajos de la escuela, porque les puede leer lo que les pide la maestra de tarea, también le puede leer la Biblia a su madre. Pedro, por su parte, deseaba conocer el abecedario. Manuel y Jorge anhelaban leer textos sobre ganadería, ocupación a la cual se dedican. Gerardo canta en "karaoke"; sin embargo, quiere leer las letras que ya se sabe por la costumbre de escucharlas repetidamente.

El aprendizaje se generó de acuerdo con el interés de cada uno; su realidad, en este sentido, jugó un papel trascendental. No obstante, se tomó en cuenta que integran una familia, por lo cual se trabajó, con ellos, temas diferentes, respetando los saberes de cada uno. Durante las sesiones se promovió el trabajo en grupo, se generaron tareas en las que se demandaba un trabajo conjunto. La evaluación continua, por parte de los participantes, permitió el desarrollo adecuado de cada sesión.

Desde el inicio del proceso alfabetizador se promovió la siguiente idea: "Todo lo que pienses lo puedes escribir"; se resaltaron los saberes de cada uno y se les hizo ver que es posible plasmar, en papel, cualquier idea que se tenga.

Por las razones anteriores, el diálogo fue trascendental y posibilitó conocer el sentir de cada uno, en relación con las actividades desempeñadas durante el día o la semana, se valoró que cada uno es un especialista según la actividad a la cual se dedica, sea la de ama de casa, de madre, de peón, de ganadero, de mecánico u otro. 
Después de concluir el proceso, a todos se les abrieron nuevos espacios de participación, por ejemplo, Manuel y Jorge se sintieron interesados en participar en algunos programas de capacitación, tales como el curso de Manejo de pastos, el cual fue impartido en su comunidad.

La formación personal y profesional obtenida por la facilitadora, durante el proceso fue invaluable, porque al involucrarse en una tarea no por obligación, sino por convicción, creció y aprendió diariamente, junto a sus alumnos. Se generaron lazos de afecto y se identificó con las labores cotidianas, la realidad y los intereses del grupo que atendió.

\section{Convergencias}

Seguidamente, se presenta una serie de convergencias entre la teoría que expone Freire y las experiencias desarrolladas. Concretamente, se han seleccionado tres principios fundamentales de la enseñanza, entendiendo por ésta algo más que transferencia del saber, pues enseñanza es aquello que se aprende social e históricamente como hombres y mujeres, y, de esta manera, se descubre que es posible enseñar, o como lo dice Freire $(1998$, p. 44) "fue aprendiendo como percibimos que es posible enseñar". Esos principios se relacionan con las experiencias de alfabetización de personas adultas: "Enseñar exige reflexión sobre la práctica" (Freire, 1998, p. 39), “Enseñar exige alegría y esperanza" (p. 70) y “Enseñar exige respeto a los saberes de los educandos" (p. 31). Es importante anotar que la puesta en práctica de estos principios, en la pedagogía, va a permitir al educador o educadora acercarse a los ideales de dignidad, respeto y autonomía de sus estudiantes, es lo que Freire llama la coherencia entre el "saber-hacer y el saber-ser pedagógicos" (p. 12).

\section{Enseñar exige reflexión sobre la práctica}

El saber acertado, del que habla Freire, es aquel que construye o que produce el aprendiz en interacción con su profesor o profesora; no se trata de un saber exclusivo de dioses o de intelectuales. 
En la primera experiencia, se evidenció que la interacción personal permitió poner en práctica la relación dialéctica entre hacer y pensar, lo cual es fundamental en una práctica docente crítica y en la construcción de pensar acertadamente.

El pensar acertadamente, para Freire, significa superar ese saber ingenuo, que nace, de manera espontánea, en la práctica docente y que carece de rigor metódico. Es indispensable que el discurso teórico sea tan concreto que se confunda con la práctica, pues cuanta menos distancia exista entre ambos, más entendimiento se gana. Si se reflexiona sobre quiénes somos, se puede cambiar, promoverse, hacer rupturas, tomar decisiones y asumir nuevos compromisos.

Cuando nos asumimos como seres sociales, históricos y pensantes, podemos ser transformadores y creadores. De ahí, que, como lo señala la expresión: la reflexión crítica sobre la práctica es indispensable para enseñar, de no ser así, tan sólo estaríamos adiestrando al educando en una serie de habilidades.

\section{Enseñar exige alegría y esperanza}

Freire nos dice que la alegría y la esperanza son dos ingredientes fundamentales en toda práctica educativa y en el clima del espacio pedagógico. Para él, la esperanza implica que los profesores y los estudiantes aprendan, enseñen y produzcan juntos y juntas. Considera que la esperanza es parte de la naturaleza humana.

Por tanto, estas premisas se reflejan, muy bien, en la experiencia de las estudiantesdocentes con el joven con necesidades educativas especiales, ya que tanto ellas como él compartieron y construyeron juntos una serie de estrategias para la alfabetización; se abrió, así, la esperanza y la alegría de conocer un nuevo mundo: el de la lectura y la escritura, herramientas que le permitieron a este joven acceder a otras formas de comunicación con su entorno. 
Éste es un ejemplo muy claro de optimismo al que hace referencia Freire (1998), de una esperanza real y no una esperanza vana, de "una esperanza como condimento indispensable de la experiencia histórica" (p. 71).

\section{Enseñar exige respeto a los saberes de los educandos}

Freire nos indica, con esta frase, que el educador o educadora y la escuela tienen el deber de respetar los saberes, socialmente construidos, de los educandos, los cuales son parte esencial de su vivencia comunal. Por ejemplo, los participantes de estas experiencias de alfabetización llegaron a las sesiones con intereses muy particulares que los movieron a aprender a leer y a escribir: ayudar a sus hijos en las tareas, participar de las decisiones comunales, realizar mejor sus labores de agricultura, entre otras actividades.

Freire recomienda discutir con los alfabetizandos estas realidades e integrarlas a los contenidos de los procesos de enseñanza y de aprendizaje, es decir, aprovechar, al máximo, esa experiencia de vida de los educandos. La escuela, debe ir mucho más allá de "enseñar contenidos y transferirlos a los alumnos" (Freire, 1998, p. 32), debe abrirse a la discusión de la realidad de sus educandos, intimar, como dice Freire, los saberes curriculares fundamentales con la experiencia social de las y los estudiantes. De esta manera, estaríamos humanizando el acto pedagógico, independientemente del contexto o nivel educativo en el que se lleve a cabo.

\section{Conclusión}

Las experiencias de alfabetización descritas permitieron confirmar la vigencia de los postulados pedagógicos de Pablo Freire, los cuales revalidan la importancia de la lectura y la escritura, para la liberación del ser humano, al dignificarle como persona autónoma, y capaz de ejercer su ciudadanía. Asimismo, se evidencia la necesidad de atender a las personas jóvenes y 
adultas que no han tenido la oportunidad de aprender a leer y escribir y, por tanto, se encuentran marginadas dentro de la sociedad actual.

Por otra parte, la vivencia de alfabetizar a personas adultas impacta la formación de quienes se preparan para ejercer la docencia, y desarrolla su sensibilidad y compromiso social. Esto tiene que ver con su responsabilidad al promover el aprendizaje de la lectura y la escritura de los niños y las niñas en la escuela primaria, procurando que todos y todas reciban lo que les corresponde por derecho, y evitar, así, que se conviertan en personas adultas no alfabetizadas.

Las demandas de la sociedad actual imponen la urgencia de una coordinación de esfuerzos entre instituciones para optimizar el uso de los recursos, y vemos que esto sí es posible; tal y como se describe en la experiencia relatada, en la que integrantes del ICE y de la UNA compartieron el reto en la alfabetización de personas jóvenes y adultas.

\section{Referencias}

Freire, P. (1998a). Pedagogía de la autonomía. Madrid: Siglo XXI Editores.

\section{Otras fuentes consultadas}

Freire, P. (1986). Hacia una pedagogía de la pregunta. Buenos Aires: Ediciones La Aurora.

Freire, P. (1992). Pedagogía del oprimido. Bogotá: Editorial América Latina.

Freire, P. (1998b). Pedagogía de la esperanza. Madrid: Siglo XXI Editores.

Freire, P. (1998c). Política y educación. México: Siglo XXI Editores.

Freire, P. (2006). Pedagogía de la indignación. Buenos Aires: Ediciones Morata.

Freire, P. (2007). Cartas a quien pretende enseñar. Buenos Aires: Siglo XXI Editores. 